\title{
Perfil clínico-epidemiológico dos pacientes internados na clínica pediátrica em um hospital universitário
}

\section{Profile clinic-epidemiological of hospitalized patients in clinic pediatric in a university hospital}

\author{
José Sávio Menezes Parenteํ. Francisco Ranilson Alves Silva². \\ 1 Residência Médica em Pediatria pelo Hospital Universitário Walter Cantídio (HUWC), Universidade Federal do Ceará (UFC), \\ Fortaleza, Ceará, Brasil. 2 Mestre em Saúde da Criança e do Adolescente, Médico-Pediatra do Hospital Universitário Walter \\ Cantídio (HUWC), Universidade Federal do Ceará (UFC), Fortaleza, Ceará, Brasil.
}

\section{RESUMO}

Introdução: a infância é um período de grande importância na formação do ser humano, logo, um episódio de doença pode interromper esse processo, além de interferir na estrutura familiar e gerar ônus ao sistema de saúde. A epidemiologia e a morbidade atuam como importantes instrumentos, pois promovem o conhecimento em saúde e auxiliam na distribuição dos recursos, alcançando os vários níveis administrativos. Porém, estudos que envolvem o perfil de internações pediátricas ainda são escassos. O estudo tem como objetivo delinear perfil epidemiológico e clínico dos pacientes internados na enfermaria de pediatria. Metodologia: estudo realizado na enfermaria de pediatria do Hospital Universitário Walter Cantídio (HUWC) com amostra de 119 registros, referentes aos meses de agosto a dezembro de 2015. Resultados: a idade média é de 8,6 anos, sendo que no sexo feminino houve predomínio na faixa da adolescência. Os 3 sistemas mais acometidos foram: sistema digestivo, sistema endócrino e sistema respiratório. A permanência hospitalar média foi de 10,09 dias. Condições crônicas foram encontradas em $48,1 \%$ dos registros, sendo o Diabetes Mellitus a mais comum. 25,2\% dos casos possuíam internamentos prévios, em sua grande maioria portadores de patologias crônicas. A grande maioria das altas ocorreram para domić́lio. Discussão e Conclusão: o estudo demonstra particularidades da enfermaria de pediatria do HUWC, sendo possível guiar o ensino de residentes e estudantes, além de auxiliar na formação de políticas, melhoria do atendimento e preparo da equipe ligada aos cuidados com o paciente.

Palavras-chave: Epidemiologia. Pediatria. Internação hospitalar.

\section{ABSTRACT}

Introduction: childhood is a very important period in the formation of the human being, thus, an episode of illness can interrupt this process, in addition to interfering in family structure and generate cost to the health system. The epidemiology and morbidity act as important tools for promoting health knowledge and assist in the distribution of resources, reaching the various administrative levels. However, studies involving the profile of pediatric admissions are still scarce. The study aims to delineate clinical and epidemiological profile of patients admitted to the pediatric ward. Methodology: a study conducted in HUWC's pediatric ward with a sample of 119 records for the months of August to December 2015. Results: the mean age of 8.6 years, and in females predominated in the teenage group. The 3 most affected systems were: digestive system, endocrine system and respiratory system. The average hospital stay was 10.09 days. Chronic conditions were found in $48.1 \%$ of the records, and diabetes mellitus was the most common. $25.2 \%$ of cases had previous admissions, mostly with chronic diseases. The vast majority of discharge occurred to home Discussion and Conclusion: the study shows particularities of HUWC's pediatric ward, and can guide the teaching of residents and students, and assist in the formation of policies, improvement of customer service and preparation of staff connected to patient care.

Keywords: Epidemiology. Pediatrics. Hospital internment.

Autor correspondente: José Sávio Menezes Parente, Rua Professor Manuel Pinto Filho, 242, Alto do Cristo, Sobral, Ceará. CEP: 62020-535.Telefone: +55 88 99713-2742/85 98756-1234. E-mail: jsaviomp@gmail.com

Conflito de interesses: Não há qualquer conflito de interesses por parte de qualquer um dos autores.

Recebido em: 03 Mar 2016; Revisado em: 04 Out 2016; Aceito em: 05 Out 2016. 


\section{INTRODUÇÃO}

O processo de crescimento e de desenvolvimento tem na infância um período de extrema importância para os aspectos biológicos, psicossociais e cognitivos. Um episódio de doença neste período pode significar um trauma, bem como atraso ou mesmo interrupção nesse processo. ${ }^{1}$ Além disso, o internamento hospitalar da criança, na maioria das vezes, representa um problema complexo na estrutura familiar, além de causar um ônus financeiro ao sistema de saúde, sendo ele público ou privado. ${ }^{2}$

Sobre a situação da saúde da criança em nosso meio, aponta-se para a necessidade de novas estratégias, as quais possam estar relacionadas ao cuidado com a criança e com a formação de novos profissionais, em destaque pediatras, que possam dispensar os cuidados necessários ao ser humano em crescimento e desenvolvimento, com todas as suas particularidades. É importante que tais estratégias sejam implementadas nos serviços de saúde e no campo de produção científica. $^{3}$

A epidemiologia serve como instrumento de conhecimento, de gestão e de transformação. Ela pode descrever a distribuição e a magnitude dos problemas de saúde, proporcionar dados especiais para o planejamento e avaliação de ações, identificar fatores etiológicos na gênese das enfermidades, auxiliar a tomada de decisão, identificar o impacto das ações implementadas, além de ter seu valor formativo e processual. ${ }^{4}$ Logo, o conhecimento da epidemiologia é de extrema importância em todas as áreas médicas, pois fornece subsídios de avaliação e de organização para programas de tratamento e campanhas de prevenção. ${ }^{5}$

Os estudos sobre internações e morbidades contribuem com dados importantes para traçar o perfil de pacientes e de doenças, além de servir de ferramenta para a planificação de serviços de saúde, a fim de diminuir a mortalidade infantil. ${ }^{2}$ Porém, grandes levantamentos epidemiológicos são encontrados em poucos estudos realizados nos países em desenvolvimento. ${ }^{6}$ Alguns estudos têm sido publicados relatando a experiência da realização de um perfil epidemiológico de algumas enfermarias e até mesmo de UTI's de estados brasileiros, como exemplo Rio de Janeiro (RJ), Florianópolis (SC), Porto Alegre (RS), Pelotas (RS), Joinville (SC), Campo Grande (MS), Teresina (PI) e Ribeirão Preto (SP), porém a disponibilidade de informação, mesmo no campo da produção científica, sobre o perfil e as principais demandas das internações pediátricas, ainda é escassa, necessitando de futuras pesquisas. ${ }^{3}$

No intuito de aperfeiçoar o manejo dessas condições, fazse necessário conhecer especificamente as etiologias mais comumente envolvidas em hospitalizações pediátricas. A partir das informações obtidas, pode-se desencadear uma sequência de medidas preventivas em saúde pública, podendo ser citado o treinamento de profissionais, a criação de programas municipais de doenças crônicas, a padronização dos atendimentos em unidades primárias e secundárias e a criação de protocolos para as doenças mais comuns. ${ }^{7}$ Além dessas medidas, o conhecimento auxilia na racionalização de instrumentos da assistência médica, evita a subutilização e o desperdício, atendendo mais eficazmente a população. ${ }^{8}$

O auxílio dessas medidas tem alcance em todos os níveis administrativos (Federal, Estadual e Municipal), além de amparar técnico-cientificamente melhor diversos profissionais que atuam diariamente com pacientes pertencentes à faixa pediátrica e diversas particularidades que envolvem os cuidados com a criança, em um momento de delicadeza, tanto para esta quanto para sua família, que é o período da internação hospitalar.

Entre 2002 e 2006, 17,2\% dos internamentos no Brasil foram de crianças com 0 a 9 anos de idade, predominando no sexo masculino. ${ }^{9}$ Em 2008, 9,6\% das internações hospitalares no Brasil foram de adolescentes. Esse número se compara ao de outras faixas etárias ocorridas no mesmo ano, sendo 16,4\% para menores de 11 anos e 15,9\% para idosos acima de 65 anos, o que constitui uma taxa expressiva e importante de hospitalizações do país. Ocorreu variância entre a segunda causa mais comum de internamento, no Norte e Nordeste as doenças infecciosas e parasitárias ficaram em segundo lugar, já no Centro-Oeste, Sul e Sudeste, as doenças ligadas ao aparelho geniturinário. ${ }^{10}$

Várias intervenções em saúde foram utilizadas afim de diminuir a morbimortalidade da população. Entre essas intervenções, pode-se pontuar melhor atenção pré-natal, melhora do suporte nutricional, promoção do aleitamento materno, melhora do saneamento e instalação dos programas de imunização. ${ }^{11}$

Porém, mesmo com o declínio progressivo da mortalidade infantil, não ocorreu a mesma evolução com o número de internações em pediatria. ${ }^{9}$ Percebe-se, também, um concomitante aumento das doenças crônicas na faixa pediátrica, além de uma tendência de aceleração na transição das doenças agudas para as doenças crônicas nos próximos anos. Fato que pode mudar gradativamente o perfil de internamentos e patologias nos hospitais públicos e privados, além de originar uma nova forma de cuidados com a saúde da criança. ${ }^{12}$

Conhecer o perfil dos pacientes internados em uma enfermaria pode gerar uma série de benefícios à instituição, ao profissional e ao usuário. Além disso, pode servir como comparativo com os dados nacionais e de outras regiões, afim de reconhecer uma particularidade regional nos casos encontrados e uma possível padronização ou individualização de condutas, bem como planejamento e estruturação do serviço.

O estudo tem o objetivo de delinear perfil epidemiológico e clínico dos pacientes internados na enfermaria de pediatria do Hospital Universitário Walter Cantídio (HUWC), além de determinar o tempo de permanência das internações, analisar a idade dos pacientes internados, caracterizar a distribuição de patologias agudas e crônicas e avaliar as principais patologias dos pacientes internados. 


\section{METODOLOGIA}

Trata-se de trabalho retrospectivo desenvolvido na enfermaria de Pediatria do HUWC vinculado à Universidade Federal do Ceará (UFC). Foi obtido consentimento prévio da chefia médica do serviço de pediatria.

Para preenchimento do instrumento, foi utilizado a $2^{\mathrm{a}}$ via dos relatórios de alta dos internamentos na enfermaria. Os dados disponíveis referem-se aos meses de agosto/2015 a dezembro/2015. Foram incluídos todos os registros que demonstravam condução clínica pelo médico-pediatra e pelo residente de pediatria. Registros que apresentavam como causa de internação patologias de condução exclusivamente cirúrgica, sem ter havido condução clínica pelo médicopediatra, foram excluídos no estudo. Obteve-se um total de 119 registros, após a análise dos critérios.

Nos dados analisados, procuramos por informações de idade e sexo. A classificação da idade foi realizada de forma tradicional, sendo dividida em neonatos (menores de 30 dias), lactentes (menores de 2 anos), pré-escolares (2 anos à 6 anos incompletos), escolares (6 anos à 10 anos incompletos) e adolescentes (10 anos à 18 anos incompletos).

Foram analisadas informações referentes a condições clínicas com perfil de cronicidade, classificando-se como aguda ou crônica. Considerou-se como doença crônica crianças portadoras de malformações congênitas, doenças genéticas ou condições clínicas com perfil de cronicidade. Para patologias que não possuíam tempo específico determinado para classificação de cronicidade, utilizou-se o critério de Vargas que considera qualquer condição com tempo de duração maior que seis meses como crônica. ${ }^{13}$ Este critério também foi utilizado por Duarte et al, 2012 em um outro estudo envolvendo o perfil de pacientes pediátricos nos hospitais do Rio de Janeiro. Foi avaliado a existência de internamentos

Gráfico 1. Número de pacientes por idade divididos por sexo.

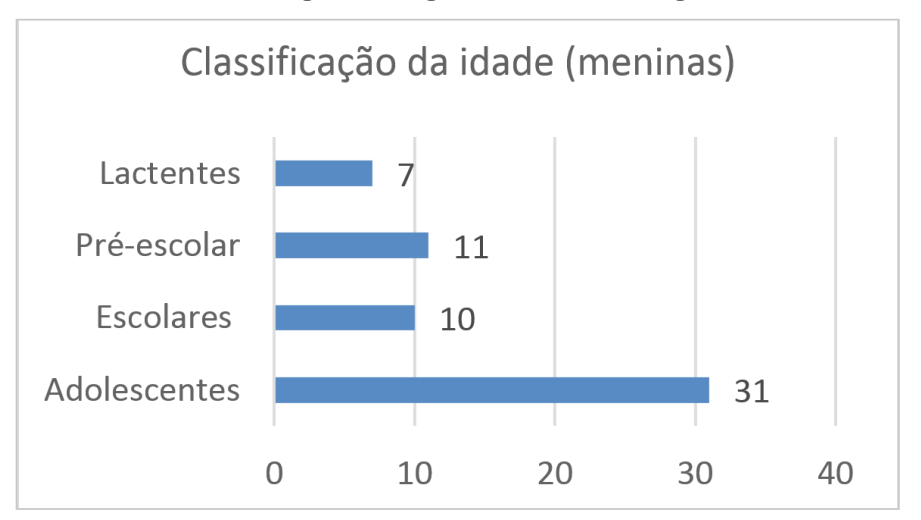

Quanto ao diagnóstico, encontramos que doenças do aparelho digestivo (K00-K93.8) foram a causa mais comum de internações com 17,6\%. Constipação crônica, doença celíaca e alergia à proteína do leite de vaca (APLV) foram as 3 principais etiologias encontradas. Doenças endócrinas, prévios, não foi considerado o número de internamentos prévios.

Analisou-se as condições de alta e diagnóstica. As condições de alta foram divididas em cura/melhorado, transferência, acompanhamento ambulatorial e óbito. Foi utilizado o diagnóstico no momento de alta e, quando na presença de dois ou mais, considerou-se o principal diagnóstico como motivo de internação, dividindo-os em grupos conforme classificação do CID-10 (Classificação Internacional de Doenças).

Aprovado pelo comitê de ética da Associação Brasileira de Odontologia $(\mathrm{ABO})$ - secção Ceará (número CAAE 54643116.3.0000.5034).

Os dados foram reunidos e analisados através do Microsoft Excel, tendo seus principais resultados apresentados abaixo.

\section{RESULTADOS}

Dos 119 registros analisados, 17 (14,28\%) obtiveram alta em agosto, 28 (23,52\%) em setembro, $36(30,28 \%)$ em outubro, $28(23,52 \%)$ em novembro e $10(8,4 \%)$ em dezembro. Quanto ao sexo, $60(50,4 \%)$ foram crianças do sexo masculino e 59 $(49,6 \%)$ do sexo feminino.

Referente à distribuição por faixa etária, encontramos que $24(20,1 \%)$ eram lactentes, $27(22,7 \%)$ eram pré-escolares, $22(18,5 \%)$ eram escolares e $46(38,7 \%)$ eram adolescentes. Não houve internamentos de crianças menores de 1 mês. A idade média das crianças internadas foi 8,6 anos e as idades variaram de 1 mês e 17 dias até 17 anos e 8 meses.

Quando analisamos a classificação etária levando-se em consideração o sexo, percebemos que no sexo feminino ocorreu predomínio na adolescência com 31 (52,54\%) de seu total. Diferentemente, em sexo masculino, não ocorreu uma prevalência de faixa etária conforme o Gráfico 1.

\section{Classificação da idade (sexo masculino)}

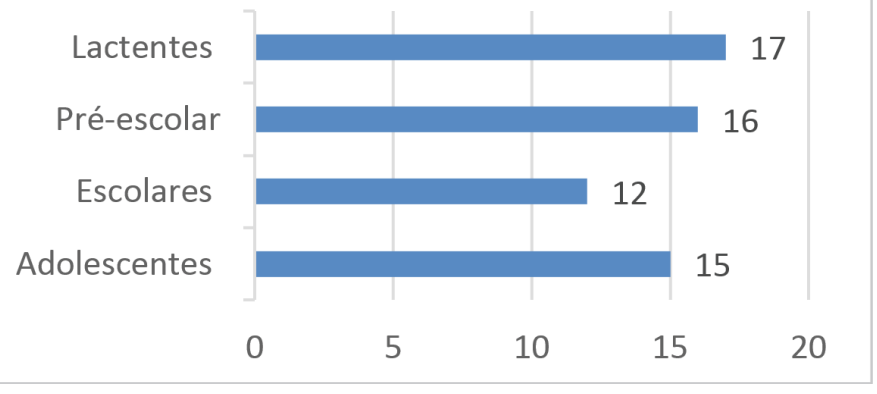

nutricionais e metabólicas (E00-E90) estavam em segundo lugar com 16,8\% dos registros, apresentando Diabetes Melitus tipo 1 como principal etiologia, seguida de hipoglicemia não especificada. Doenças do aparelho respiratório (J00-J99.8) e doenças do aparelho geniturinário (N00-N99.9) estavam na 
terceira condição mais prevalente, ambas com 12,6\% dos motivos de internação. As etiologias respiratórias mais comuns foram pneumonia, broquiolite viral aguda (BVA) e asma, em ordem de prevalência. A etiologia do aparelho geniturinário mais encontrada foi a síndrome nefrítica. Dando continuidade, temos as doenças do sistema osteoarticular e do tecido conjuntivo (M00-M99.9) com 9,2\% sendo febre reumática a etiologia mais comum e as doenças do sangue e dos órgãos hematopoiéticos (D50-D89.9) com 8,4\% apresentando a anemia falciforme a causa mais comum.

Quando levado em conta sexo feminino, o grupo de doenças mais comum foram as doenças do aparelho digestivo com $23,72 \%$, seguido por doenças endócrinas, nutricionais e metabólicas e por doenças do aparelho geniturinário ambas com $15,25 \%$. No sexo masculino, as doenças do aparelho respiratório e as doenças endócrinas, nutricionais e metabólicas ficaram em primeiro lugar, ambas com 18,33\%, em segundo lugar as doenças do aparelho digestivo com 13,33\% e em terceiro lugar as doenças do sistemas oosteoarticular e do tecido conjutivo e as doenças do aparelho geniturinário, ambas com $11,66 \%$.

O tempo permanência do internamento variou de 1 dia até 70 dias (Gráfico 2). A média de duração da internação foi de 10,09 dias. Os diagnósticos que geraram tempo de internamento mais curto foram: asma, anemia falciforme e síndrome genética não especificada. O internamento mais prolongado teve como diagnóstico lúpus eritematoso sistêmico.

Gráfico 2. Relação do tempo de internamento com a idade do paciente.

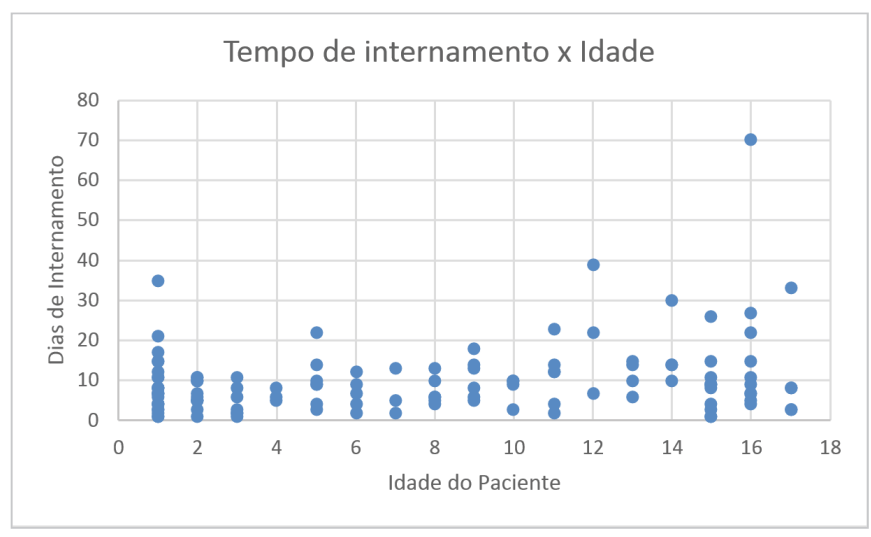

As doenças crônicas foram encontradas em 58 (48,1\%) dos registros. diabetes mellitus foi a principal etiologia encontrada, presente em 13 deles. Dos pacientes com doenças crônicas, $50 \%$ deles já possuíam história prévia de internamento. A média da duração do internamento foi 11,1 dias para as condições crônicas.

De todos os registros, 89 (74,8\%) não possuíam história prévia de internamento hospitalar, sendo seu primeiro internamento. Os outros $30(25,2 \%)$ possuíam história prévia de internação, $29(96,6 \%)$ deles eram pacientes considerados com doenças crônicas, somente $1(3,4 \%)$ foi classificado com patologia aguda. Dos registros com história de outros internamentos prévios, 11 deles estavam no grupo das doenças endócrinas, nutricionais e metabólicas, 8 deles estavam em grupo de doenças do sangue e dos órgãos hematopoéticos e 5 deles no grupo de doenças do aparelho digestivo. A principal causa de reinternação entre as doenças endócrinas era diabetes mellitus descompensado.

Considerando os motivos de alta, nenhuma das internações apresentou o óbito como desfecho final. A grande maioria das altas da enfermaria ocorreram para o domicílio (95,8\%), destes $57,8 \%$ tiveram retorno agendado no ambulatório de pediatria. Em alguns poucos casos, para dar continuidade ao tratamento, foi necessário a transferência do paciente para outro hospital (4,2\%).

\section{DISCUSSÃO E CONCLUSÃO}

O estudo possibilitou conhecer algumas características ainda pouco estudadas das internações que ocorrem na enfermaria de pediatria do HUWC, além de ser possível analisar algumas variáveis, podendo demonstrar peculiaridades no perfil das internações pediátricas.

Na amostra estudada, encontramos uma quantidade equivalente entre as internações quando considerado sexo masculino e sexo feminino. Considerando as diferentes faixas etárias, não ocorreu prevalência específica no número total, porém, quando considerado a divisão por sexo, o sexo feminino apresentou uma prevalência no período da adolescência, envolvendo $52,54 \%$ do total de registros. O mesmo resultado não ocorreu no sexo masculino, tendo sua maioria pertencentes a faixas etárias menores de 10 anos com somente $25 \%$ pertencendo à adolescência. Esse resultado fortalece a existência de um predomínio do sexo masculino nas internações pediátricas abaixo de 10 anos e o predomínio do sexo feminino nas internações pediátricas acima de 10 anos.

As doenças do aparelho digestivo foram a principal causa de internamento, o que diferencia do demonstrado nacionalmente entre 2002 e 2006 quando essas causas se encontravam em $4^{\circ}$ lugar. ${ }^{10}$ As doenças do aparelho respiratório, consideradas as principais causas de internamento, ${ }^{10}$ ficaram em terceiro lugar como causa mais comum nos internamentos durante período estudado. As doenças endócrinas, nutricionais e metabólicas se mostraram como importante causa de internamento, como causa para esse resultado devemos levar em consideração a presença importante de um serviço de endocrinologia pediátrica com referência local, além da presença de residência médica em endocrinologia pediátrica. Tratando-se de um hospital universitário, saber que as patologias do aparelho digestório, endócrino e respiratório são as principais causas de internação hospitalar, pode direcionar o ensino para médicos residentes de pediatria e estudantes de medicina para essas condições mais prevalentes.

O tempo de permanência hospitalar variou de 1 a 70 dias, porém o maior tempo de permanência encontrado parece ser um caso isolado, visto que ocorreu uma única vez. A média de tempo de permanência foi de 10,09 dias, considerando que entre 2002 e 2006 o tempo de permanência das internações pediátricas no Brasil variou entre 3,4 e 7,0 dias, ${ }^{10}$ temos que a média de permanência no HUWC é superior ao maior 
tempo de permanência apresentado há cerca de uma década atrás. Esse maior período de permanência talvez possa ser justificado pelo fato de quase $50 \%$ dos internamentos serem de pacientes com condições consideradas crônicas, o que acarreta em condições mais complexas e que necessitam de maiores cuidados, o que pode resultar no prolongamento da internação. Referente à prevalência de patologias crônicas, Duarte, 2012 apresentou um achado semelhante com 47,6\% das crianças sendo portadoras de doença crônica.

Dos registros que relatavam internações prévias, quase $100 \%$ delas ocorreram em pacientes com condições crônicas, esse fato pode confirmar que pacientes com essas condições são mais vulneráveis a complicações e a intercorrências, nos

\section{REFERÊNCIAS}

1. Bortolote GS, Brêtas JR. O ambiente estimulador ao desenvolvimento da criança hospitalizada. Rev Esc Enferm USP. 2008;42(3):422-9.

2. Granzotto JA, Fonseca SS, Steffen MS, Machado MM, Roncaglio $\mathrm{R}$, Lima DP, et al. Fatores relacionados à internação pediátrica em um hospital universitário da Região Sul do Brasil. Pediatria (São Paulo). 2010;32(1): 15-19.

3. Duarte JG, Gomes SC, Pinto MT, Gomes MA. Perfil dos pacientes internados em serviços de pediatria no município do Rio de Janeiro: mudamos? Physis. 2012;22(1):199-214.

4. Rouquayrol MZ, Silva ML. A epidemiologia na organização dos serviços de saúde. Sitientibus. 1998;19:55-67.

5. Coutinho BB, Balbuena MB, Anbar RA, Anbar RA, Almeida KG, Almeida PY. Perfil epidemiológico de pacientes internados na enfermaria de queimados da Associação Beneficente de Campo Grande Santa Casa/MS. Rev Bras Cir Plast. 2010;25(4): 600-603.

6. Einloft PR, Garcia PC, Piva JP, Bruno F, Kipper DJ, Fiori RM. Perfil epidemiológico de dezesseis anos de uma unidade de terapia intensiva pediátrica. Rev Saude Publica. 2002;36(6):728-33.

7. Veras TN, Sandim G, Mundim K, Petrauskas R, Cardoso G, levando a refletir sobre a necessidade de maiores investimentos em recursos, visto que pacientes portadores de patologias crônicas geralmente necessitam de maiores cuidados.

A ausência de óbitos e a alta domiciliar ocorrendo na grande maioria das internações demonstram a existência da boa condução clínica dos pacientes que passaram pela enfermaria do HUWC.

O estudo demonstra particularidades e características próprias de pacientes que recebem assistência médica na enfermaria do HUWC, o que pode ajudar na formação de políticas, na melhoria do atendimento e no preparo da equipe que está diretamente ligada nos cuidados com o paciente.

D`Agostin J. Perfil epidemiológico de pacientes pediátricos internados com pneumonia. Sci Med (Porto Alegre). 2010;20(4):27781.

8. Rocha JS. Utilização de leitos hospitalares em Ribeirão Preto, São Paulo (Brasil). Rev Saude Publica. São Paulo, 1975;9(4):477-93.

9. Ferrer AP. Estudo das causas de internação hospitalar das crianças de 0 a 9 anos de idade no município de São Paulo [dissertação]. São Paulo: Faculdade de Medicina da Universidade de São Paulo; 2009. 144p.

10. Dornellas PM. Adolescentes no Brasil: internações hospitalares no Sistema Único de Saúde [dissertação]. Paraná: Universidade Estadual de Londrina; 2011. 134p.

11. Victora CG. Intervenções para reduzir a mortalidade infantil préescolar e materna no Brasil. Rev Bras Epidemiol. 2001;4(1):3-69.

12. Vieira MA, Lima RA. Crianças e adolescentes com doença crônica: convivendo com mudanças. Rev Lat Am Enfermagem. 2002;10(4):552-60.

13. Vargas N, Arredondo O, Ilabaca G, Maturana A, Ortuvia G. Enfermedades crónicas en pacientes pediátricos hospitalizados: frecuencia y tipo de enfermedad. Rev Chil Pediatr. 1994;65(5) 64-7.

\section{Como citar:}

Parente JS, Silva FR. Perfil clínico-epidemiológico dos pacientes internados na clínica pediátrica em um hospital universitário. Rev Med UFC. 2017 jan-abr;57(1):10-14. 